\title{
Multiscale Modeling of Metallic Materials Containing Embedded Particles
}

\author{
D. R. Phillips* \\ NASA Langley Research Center, Hampton, VA, 23681-2199 \\ E. Iesulauro ${ }^{\dagger}$ \\ Cornell University, Ithaca, NY, 14853 \\ and \\ E. H. Glaessgen \\ NASA Langley Research Center, Hampton, VA, 23681-2199
}

\begin{abstract}
Multiscale modeling at small length scales $\left(10^{-9}\right.$ to $\left.10^{-3} \mathrm{~m}\right)$ is discussed for aluminum matrices with embedded particles. A configuration containing one particle surrounded by about 50 grains and subjected to uniform tension and lateral constraint is considered. The analyses are performed to better understand the effects of material configuration on the initiation and progression of debonding of the particles from the surrounding aluminum matrix. Configurational parameters considered include particle aspect ratio and orientation within the surrounding matrix. Both configurational parameters are shown to have a significant effect on the behavior of the materials as a whole. For elliptical particles with the major axis perpendicular to the direction of loading, a particle with a 1:1 aspect ratio completely debonds from the surrounding matrix at higher loads than particles with higher aspect ratios. As the particle major axis is aligned with the direction of the applied load, increasing amounts of load are required to completely debond the particles.
\end{abstract}

\section{Introduction}

$\mathrm{M}$ ULTISCALE modeling provides an efficient means of interrogating deformation and fracture of metallic materials from the micro- to the nano-scales. Cohesive zone models are an important technique used in multiscale modeling of grain-scale events and can be used to study grain boundary decohesion of metallic materials under loading. Similar grain-scale simulations can be used to quantify the effect of particles on load transfer and fracture initiation within the metallic materials.

Determination of the interaction (particularly, the load transfer) between particles and a surrounding metallic microstructure (Figure 1) is a topic of considerable attention within the materials processing community. Deformation and fracture at length scales such as those associated with the particle may not be adequately modeled by traditional continuum mechanics-based concepts. Rather, the atomic-level events related to bond breakage, dislocation motion, and void nucleation must be understood and then used to predict debonding at the grain scale. This process requires an understanding of material behavior at the atomic level as well as development of means to bridge length scales.

In this paper, multiscale modeling of deformation and fracture of metallic materials with embedded particles will be discussed. Cohesive

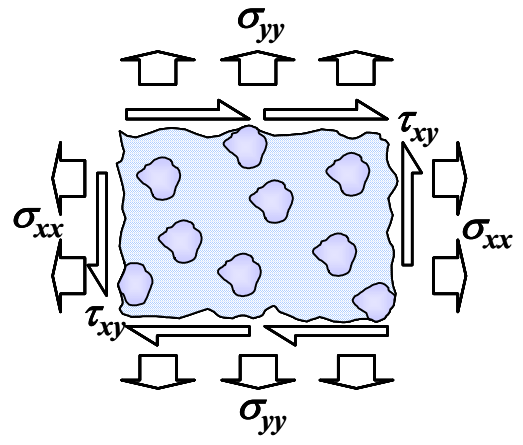

Figure 1: Particles embedded in a metallic matrix subjected to far-field loading

\footnotetext{
* Aerospace Engineer, Lockheed Martin Space Operations, NASA Langley/240, Member AIAA.

${ }^{\dagger}$ Graduate Student, Cornell Fracture Group, 640 Rhodes Hall, Member AIAA.

* Aerospace Engineer, Analytical and Computational Methods Branch, NASA Langley/240, Senior Member AIAA.
} 
zone models are used to estimate the effect of the atomic-scale events in finite element (FE) -based micromechanics models of an elliptical particle embedded within an aluminum matrix. The micromechanics model is used to simulate the debonding along the grain boundaries of the aluminum matrix and between the grains of the aluminum matrix and the particles. The effects of particle size, shape, and orientation on the debonding of particles from aluminum grains will be determined. Additionally, the effects of the debonding of the particle on load transfer and fracture of the material as a whole will be discussed.

\section{Multiscale Modeling}

Length scales on the order of $10^{-9}$ to $10^{-3} \mathrm{~m}$ dominate the problem of particle-matrix debonding. Atomistic effects dominate at the lower bound of this range, while traditional continuum assumptions are valid near the higher bound. In the present analysis, cohesive zone models $(\mathrm{CZM})^{1}$, which approximate the interfacial atomic tractiondisplacement relationship using bulk material properties, are used to model the response along the grain boundaries and between the particles and the grains of the aluminum matrix. Cohesive zone models assume cohesive interactions among the grains of a material and permit the appearance of fracture surfaces in a continuum. ${ }^{2}$ One popular cohesive zone model is attributed to Tvergaard and Hutchinson ${ }^{1}$, where the normal and shear components of the traction and displacement are combined into single measures, $\tau$ and $\lambda$, respectively, so that the responses are coupled. ${ }^{3}$ The coupled cohesive zone model (CCZM) given in reference 3 defines a traction potential,

$$
\Phi\left(\delta_{n}, \delta_{t}\right)=\delta_{n}^{c} \int_{\lambda} \tau\left(\lambda^{\prime}\right) d \lambda^{\prime}
$$

where $\lambda$ is a non-dimensional measure of the relative opening and sliding displacements ( $\delta_{n}$ and $\delta_{t}$ ) as defined by

$$
\lambda=\left[\left(\frac{\delta_{n}}{\delta_{n}^{c}}\right)^{2}+\left(\frac{\delta_{t}}{\delta_{t}^{c}}\right)^{2}\right]^{1 / 2},
$$

$\delta_{n}^{c}$ and $\delta_{t}^{c}$ are the critical values for the opening and sliding modes, respectively, and the initiation of a crack is assumed to occur once $\lambda$ reaches a value of unity. The present methodology for estimating atomic scale information within the grain scale models is shown in Figure 2. Figure 2(a) represents a hypothetical traction-displacement relationship describing the atomic-level events at the grain boundary, while Figure 2(b) is the estimated response embedded within the CCZM. The traction-displacement response in Figure 2(b) is embedded within cohesive zone elements (Figure 2(c)) to model the response of the grain boundaries. The cohesive zone elements are embedded between continuum finite elements on either side of the grain boundary as shown in Figure 2(d). ${ }^{1}$ In two dimensions, the cohesive zone elements have zero width in the normal direction, i.e., the unprimed and primed nodes in Figure 2(c) are coincident.

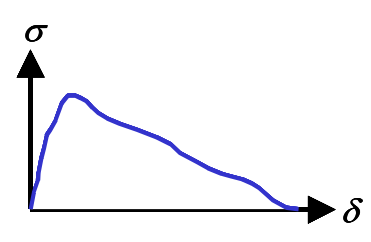

(a) Hypothetical traction-displacement relationship from MD

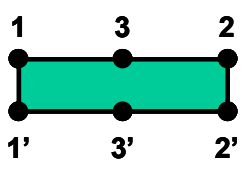

(c) Cohesive zone element (b) Estimated traction-displacement relationship in CCZM

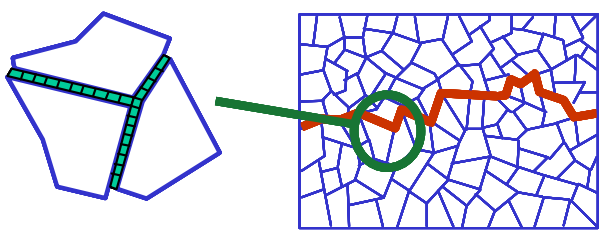

(d) Cohesive zone elements embedded in the grain boundaries of the crack path within a grain structure

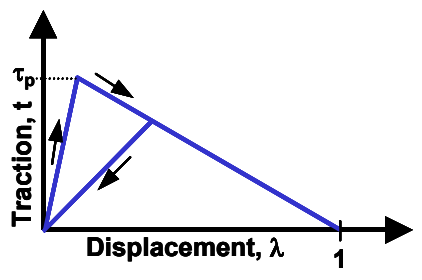

Figure 2: Implementation of the cohesive zone models. 


\section{Micro-Scale Modeling}

Voronoi tessellations are used to represent the grains of the metallic material. The tessellations are formed by first randomly distributing nuclei and connecting these nuclei via straight lines to their nearest neighbors, forming a Delaunay triangulation. ${ }^{4}$ These lines are then perpendicularly bisected to create the edges of a polygon. The resulting polygons define the Voronoi regions, the areas within which all points are closer to the nucleus of that polygon than to any other polygon nucleus. ${ }^{5}$ In the current work, Voronoi polygons represent the individual grains of a metallic material surrounding the domain of the particle (see Figure 3). Particles in this study are 1/3 to 1 times the size of the typical matrix grain size. The algorithm by which these models are generated is outlined below:

1. An elliptical particle and buffer zone are defined. Voronoi nuclei are randomly chosen such that they do not lie within the buffer zone, and a tessellation is formed (see Figure 4).

2. The Voronoi boundaries that intersect the buffer zone are clipped to the buffer, and the clipped points are connected (see Figure 5).

3. Each of the clipped points is connected to a major axis $1 / 3$ point of the elliptical particle, and then these new edges are clipped to the ellipse boundary. The points clipped to the ellipse are connected, resulting in new regions between the particle (which is distorted) and the existing regions. The curved edges of the particle are recovered by defining arc segments between the vertices. The results of these processes are shown in Figure 6.

4. Small grains that form around the ellipse are merged with neighboring grains to make a more realistic model, and the model is meshed. A finite element mesh of the model in Figure 6 is shown in Figure 7.

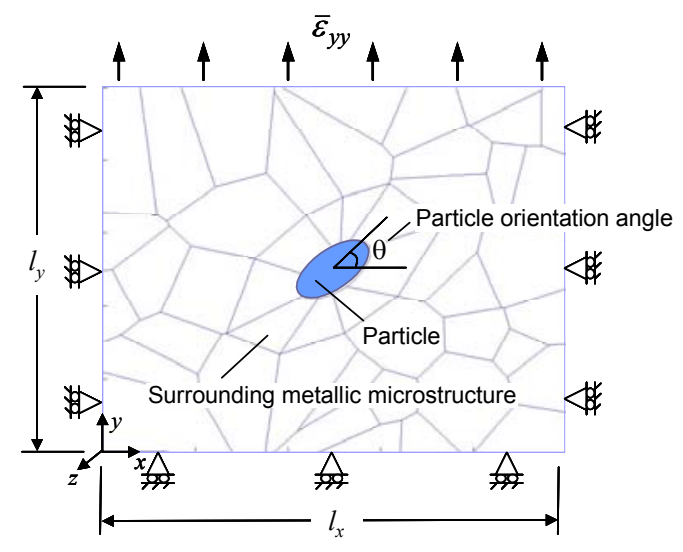

Figure 3: Model of material microstructure with a prescribed elliptical-shaped particle.

\section{Analysis}

The work in reference 6 is extended here to model load transfer and debonding of particles in an aluminum matrix. A Voronoi tessellation is generated around a prescribed elliptical-shaped particle as shown in Figure 3. The problem is assumed to be plane strain and is subjected to uniform tension and lateral constraint. The matrix is assumed to be isotropic elastic 7075-T6 aluminum, and the particle (shaded in Figure 3) is assumed to be a relatively soft isotropic elastic material. Cohesive zone elements implementing two different CCZMs are placed along the grain boundaries: one model for the aluminum-aluminum interface, and one model for the particle-aluminum interface. The material properties used for the 7075-T6 aluminum grains, the particle, and the grain boundaries are given in Table 1.

The aluminum

Table 1: Domain and grain boundary properties

matrix and particle properties include the modulus, $E$, and the Poisson's ratio, $v$. The grain boundary properties

\begin{tabular}{|c|c|c|c|c|c|}
\hline \multicolumn{3}{|c|}{ Domain Properties } & \multicolumn{3}{c|}{ Boundary Properties } \\
\hline & Aluminum & Particle & & Aluminum-Aluminum & Particle-Aluminum \\
\hline$E$ & $72 \mathrm{GPa}$ & $57 \mathrm{GPa}$ & $\tau_{p}$ & $0.5 \mathrm{GPa}$ & $0.1 \mathrm{GPa}$ \\
\hline$v$ & 0.33 & 0.32 & $k_{n}$ & $4 \times 10^{9} \mathrm{GPa}$ & $4 \times 10^{9} \mathrm{GPa}$ \\
\hline & & & $\delta_{n}{ }^{c}=\delta_{t}^{c}$ & $1 \mu \mathrm{m}$ & $1 \mu \mathrm{m}$ \\
\hline
\end{tabular}
include the peak stress, $\tau_{p}$, the initial stiffness, $k_{n}$, and the critical opening displacements normal and tangential to the interface, $\delta_{n}^{c}$ and $\delta_{t}^{c}$, respectively. The parameters that define the CCZM are chosen such that when all of the grains, including the particle, are assigned homogeneous material properties and a very small displacement is applied, load is completely transferred across the CCZM and a homogeneous stress field is obtained. The peak traction for the particle-aluminum interface is assigned a lower value than the aluminum-aluminum interface to guarantee that the particle debonds from the aluminum matrix before crack initiation occurs in the aluminum matrix. The simulation sample has dimensions $\left(l_{x}, l_{y}\right)$ along the $x$ and $y$ axes, where $l_{x}=l_{y}=1 \mathrm{~mm}$.

As the grains in the present work are all considered to be isotropic and of the same elastic properties, the grain boundaries should have no effect on the analyses before grain boundary decohesion initiates; results should agree 


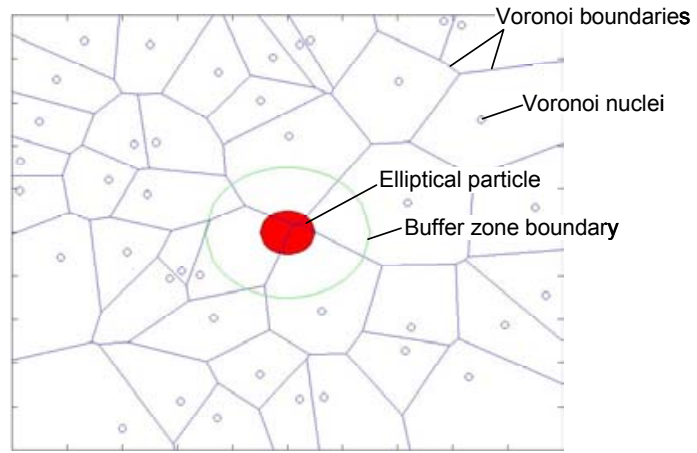

Figure 4: Voronoi diagram and overlaid particle and buffer zone.

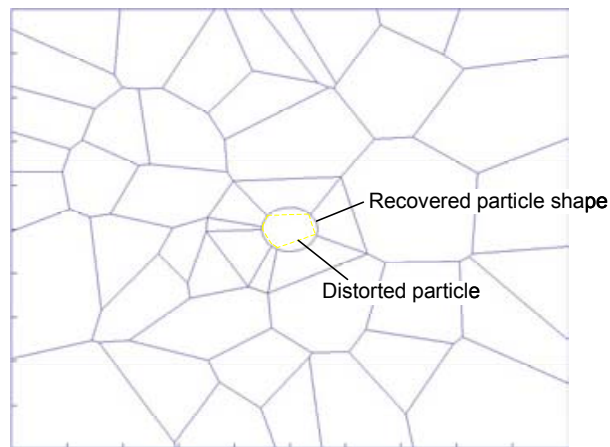

Figure 6: Voronoi diagram with curved edges of the particle recovered and underlaid distorted particle.

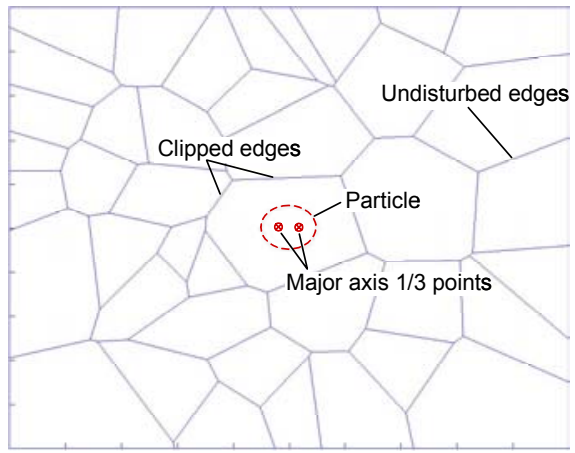

Figure 5: Voronoi diagram with clipped points connected and overlaid particle.

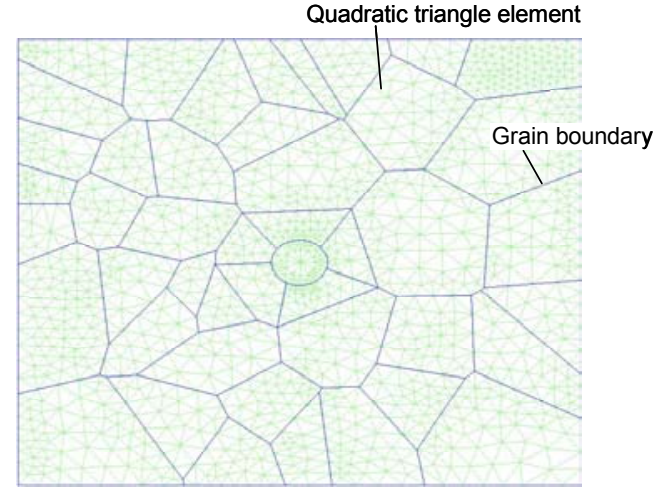

Figure 7: Finite element mesh of the model represented by Figure 6.

with those obtained by performing the analyses on particles embedded in an amorphous material. Figure 8 presents the loading-direction stress distribution at $0.1 \%$ applied strain for a circular particle embedded in (a) amorphous isotropic aluminum and (b) polycrystalline isotropic aluminum. As expected, the stress distributions in Figures 8(a) and (b) are identical, and a stress concentration is formed by the particle.

Legarth $^{6}$ studied the effects of particle aspect ratio and orientation on the onset and completion of debonding

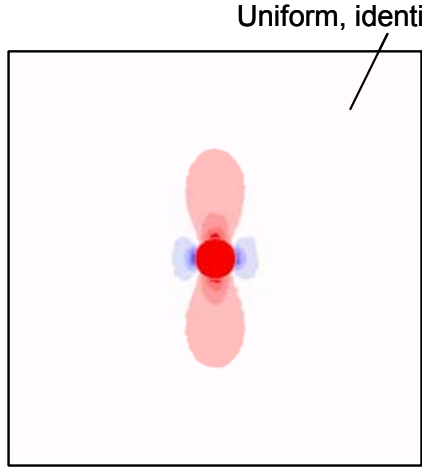

(a) Amorphous isotropic aluminum

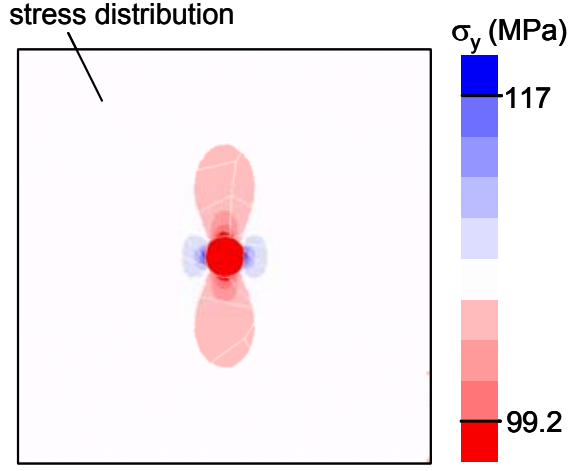

(b) Polycrystalline isotropic aluminum

Figure 8: Loading-direction stress distribution at $0.1 \%$ far-field strain for a circular particle embedded in aluminum of rigid elliptical particles from metal matrices. In the analyses, the matrix material was considered to be amorphous, and both plastic isotropic and plastic anisotropic material models were used. Coupled cohesive zone models were used to model the particle-matrix interface. Particles with $0^{\circ}$ and $90^{\circ}$ orientations and aspect ratios of 1:1, 2:1, and 4:1 were considered. Conclusions from reference 6 include:

1. The onset of debonding is nearly unaffected by the particle aspect ratio.

2. The complete debonding of the particle from the matrix requires higher strains as the major axis of the particle is aligned with the axis of load application. 
In the current study, models with elliptical particles of three different aspect ratios (AR) and four angles of orientation $(\theta)$ (given in Table 2) are considered. A summary of the problem parameters used in both reference 6 and the current work is presented in Table 3 . The boundary conditions and loading for a typical $\left(l_{x}, l_{y}\right)$ model with a particle oriented at $\theta$ degrees within a matrix tessellation are shown in Figure 3. Particles are placed in the center of the models and are 1/3 to 1 times the size of the surrounding grains. In the current work, the particle minor axis is held constant, and as the aspect ratio of the particle increases, so does its area.

Uniform far-field tension is applied in the form of $0.01 \%$ strain increments to a maximum strain of $0.5 \%$. Lateral constraint at $x=0$ and $l_{x}$ (see Figure 3 ) is assumed and will cause Table 3: Problem parameters used in reference 6 and the current work transverse stresses, $\sigma_{x}$, in the

models. The models are loaded until the peak traction, $\tau_{p}$, of the particle-aluminum interface is reached and the elliptic particles begin to debond from the surrounding matrix. The far-field strain to initiate debonding is shown in Figure 9 as a function of particle orientation angle, $\theta$, and particle aspect ratio, AR. These results indicate a small dependence (approx. 5\%) of debond initiation on both particle aspect ratio and angle of orientation with the lateral constraint boundary condition.

Loading of the elliptical particle models continues until the particles debond completely from the aluminum matrices. In this work, the particles are said to have completely debonded when tractions are no longer transferred across the particle-aluminum interface. As seen in Figure 10, increasing the angle of orientation of the 2:1 and 3:1 particles increases the strain required to completely debond the particles.

Table 4 presents the strain increase required to reach complete debonding from debonding onset for the three different aspect ratios for each of the orientation angles considered. For a 1:1 aspect ratio (i.e., a circular particle), a larger increase in strain is required to completely debond the particle once onset occurs than is required for the $2: 1$

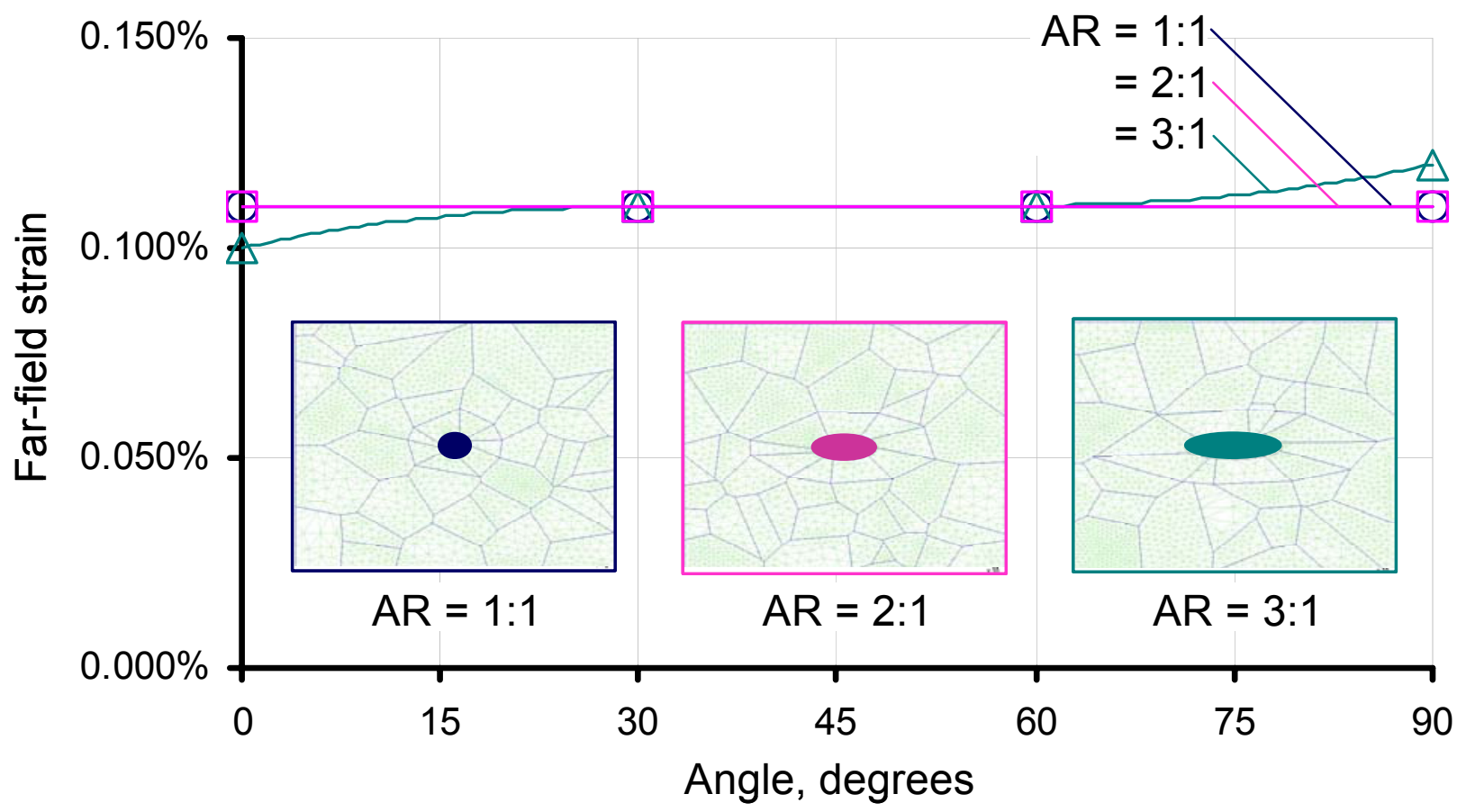

Figure 9: Far-field strain to initiate debonding (reak peak traction) for particles of three different aspect ratios embedded in an aluminum matrix. 


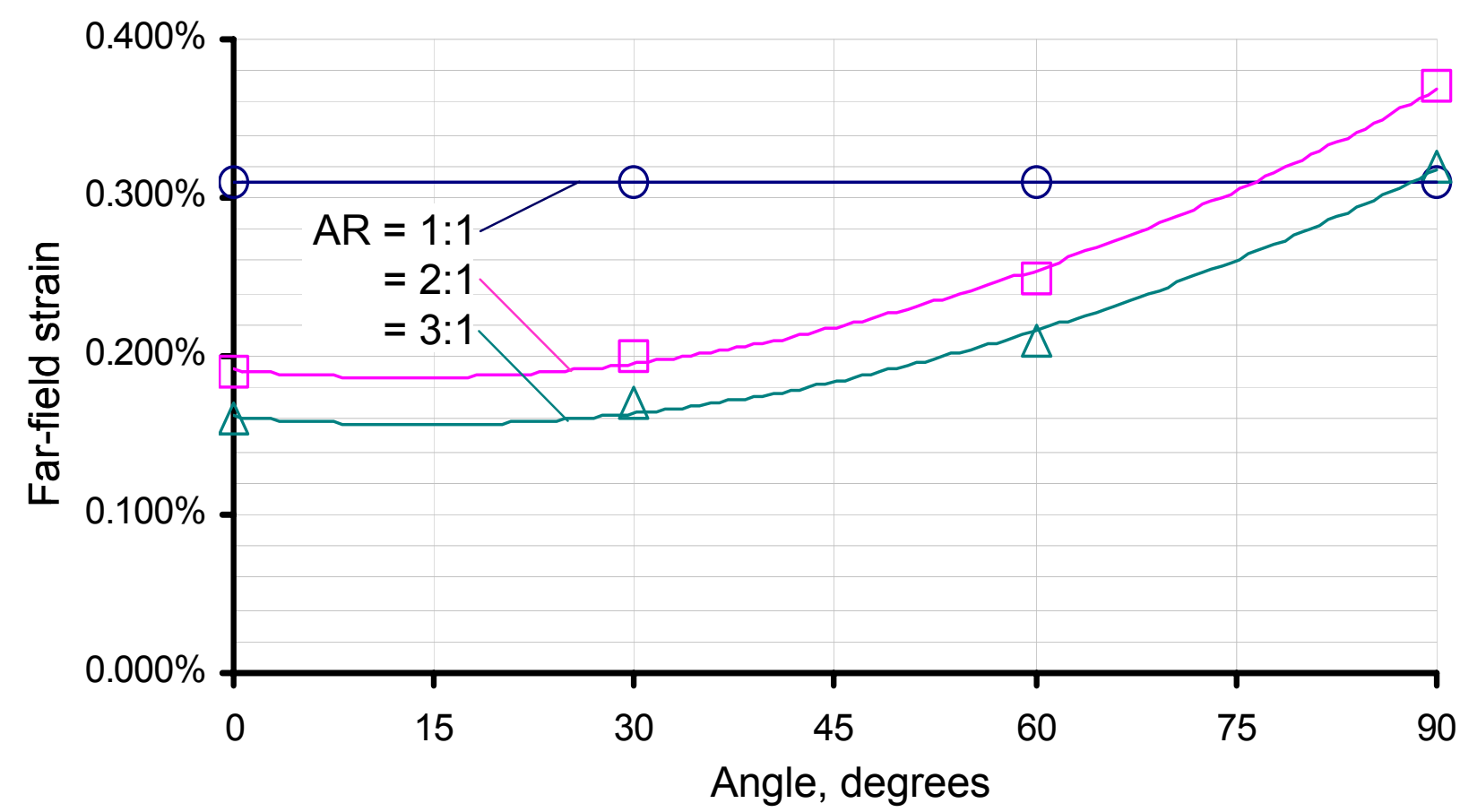

Figure 10: Far-field strain to completely debond particles of three different aspect ratios from an aluminum matrix.

and 3:1 aspect ratios. For non-circular elliptical particles, larger strain increases are required to completely debond the particles as the orientation angle aligns the particle major axis with the direction of the applied load. Figures 9 and 10 can then be used to estimate the strains at which initial and final debonding will occur. For example, particles in all three aspect ratios, when oriented at $45^{\circ}$, will begin to debond at $0.11 \%$ strain, and the $1: 1,2: 1$, and $3: 1$ particles should completely debond at $0.31 \%, 0.22 \%$, and $0.18 \%$ strain,

Table 4: Strain increment required to completely debond particles of three different aspect ratios from an aluminum matrix once onset has occurred

\begin{tabular}{|c|c|c|c|}
\hline & $1: 1$ & $2: 1$ & $3: 1$ \\
\hline $0^{\circ}$ & $0.200 \%$ & $0.080 \%$ & $0.060 \%$ \\
\hline $30^{\circ}$ & $0.200 \%$ & $0.090 \%$ & $0.060 \%$ \\
\hline $60^{\circ}$ & $0.200 \%$ & $0.140 \%$ & $0.100 \%$ \\
\hline $90^{\circ}$ & $0.200 \%$ & $0.260 \%$ & $0.200 \%$ \\
\hline
\end{tabular}
respectively.

The details of the load transfer between the particles and the surrounding aluminum matrix and the effect of the particles on the fracture of the matrix are now considered for a configuration with an elliptical particle having an aspect ratio of 2:1 and orientation of $0^{\circ}$. As shown in Figure 11, the strain in the relatively soft particle is larger than the strain in the aluminum if the local grain boundary remains connected, i.e., the peak traction of the particle-aluminum interface $\left(\tau_{p}=0.1 \mathrm{GPa}\right)$ is not surpassed. Once the peak traction is surpassed, the particle begins to debond from the surrounding aluminum (Figure 12(b)), and the load transfer across the boundary is reduced (Figure 12(a)). Stress concentrations form at the narrow edges of the elliptical particle as shown in Figure 12(a). As more strain is applied to the material, the particle continues to debond and the stresses in the material increase until the particle completely debonds from the aluminum. Further increasing the applied strain increases the stresses in the aluminum until the peak

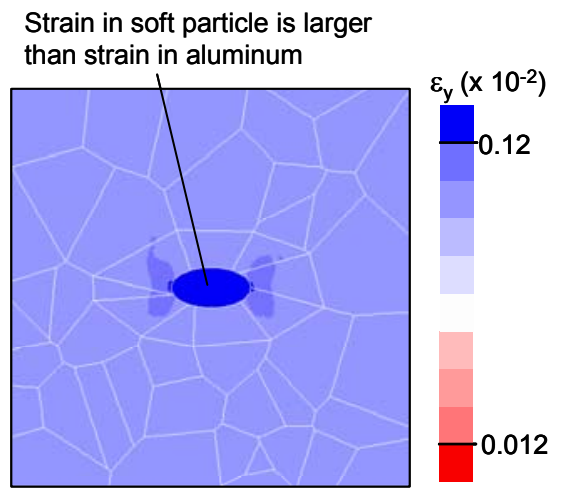

Figure 11: Loading-direction strain distribution for $0.1 \%$ far-field strain. traction of the aluminum-aluminum interface ( $\tau_{p}=0.5 \mathrm{GPa}$ ) is surpassed (at $0.20 \%$ strain), and the crack begins to grow in the aluminum grains (see Figure 13). As the crack propagates (Figure 14(b)), the stress in the material is redistributed as shown in Figure 14(a). 


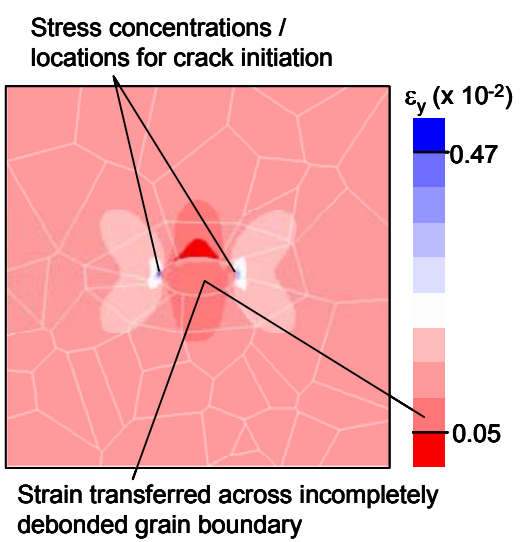

(a) Strain distribution

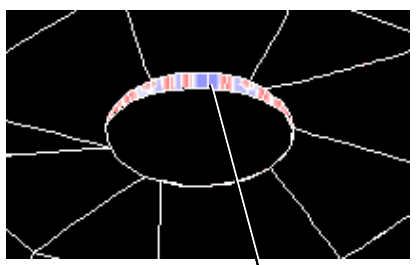

Grain boundary opening

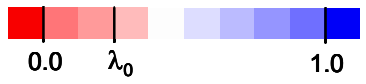

(b) Grain boundary opening

Figure 12: Loading-direction strain distribution and grain boundary opening for $0.15 \%$ far-field strain.

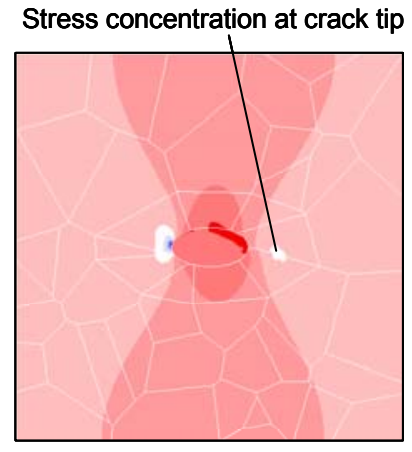

(a) Stress distribution

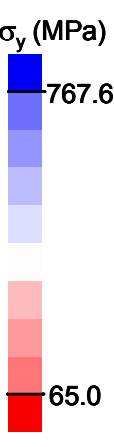

5.0

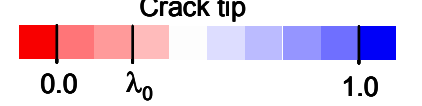

(b) Grain boundary opening

Figure 13: Loading-direction stress distribution and grain boundary opening (crack initiation) at $0.20 \%$ far-field strain.

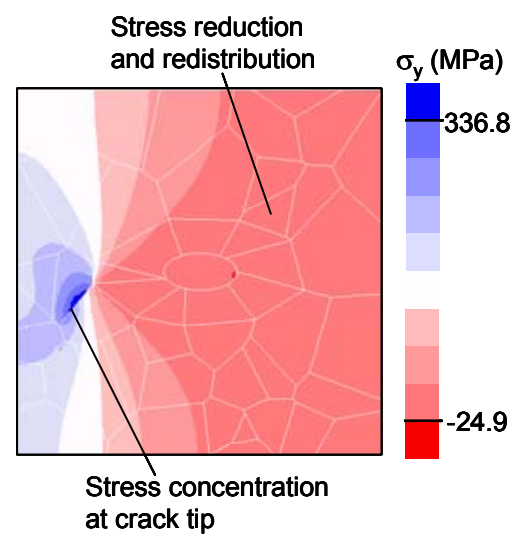

(a) Stress distribution

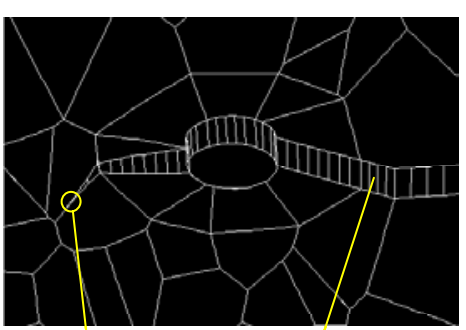

Crack tip

Through-crack

(b) Grain boundary opening

Figure 14: Loading-direction stress distribution and grain boundary opening (crack propagation) at $0.21 \%$ far-field strain.

\section{Concluding Remarks}

Multiscale modeling is discussed for isotropic aluminum matrices with embedded particles. The behavior of the grain boundaries and the particlealuminum interface is modeled using coupled cohesive zone models, which use bulk material properties to estimate an atomic-scale traction-displacement relationship.

The effects of the particle size, shape, and orientation within the aluminum matrix on debond initiation and completion, load transfer, and fracture of the matrix are studied. For the case of uniform far-field tension loading and lateral constraint boundary conditions considered, the configuration of the particle has little effect on the load at which debonding initiates. However, the configuration of the particle has significant effect on the load required for complete debonding. For elliptical particles with the major axis perpendicular to the direction of loading, a particle with a $1: 1$ aspect ratio completely debonds from the surrounding matrix at higher loads than particles with higher aspect ratios. In addition, the load required to completely debond the particle increases as its major axis is aligned with the loading direction. Debonding of the particle precipitates cracking along the grain boundaries of the aluminum matrix.

\section{Acknowledgments}

The work of Dawn Phillips is funded though contract NAS1-00135 (SAMS) with Lockheed Martin. The work of Erin Iesulauro is funded through grant NAG-102051 with Cornell University. The authors wish to thank Professor Anthony Ingraffia, and Msrs. John Emery, Mike Veilleux, and Andy Lui of the Cornell Fracture Group for their contributions to the analyses presented in this paper.

\section{References}

${ }^{1}$ Tvergaard, V. and Hutchinson, J.W., "The Relation Between Crack Growth Resistance and Fracture Process Parameters in ElasticPlastic Solids," Journal of the Mechanics and Physics of Solids, Vol. 40, pp. 1377-1397, 1992. 
${ }^{2}$ Klein, P. and Gao, H., "Crack Nucleation and Growth as Strain Localization in a Virtual-Bond Continuum," Engineering Fracture Mechanics, Vol. 61, pp. 21-48, 1998.

${ }^{3}$ Iesulauro, E., "Decohesion of Grain Boundaries in Statistical Representations of Aluminum Polycrystals," Cornell University Report No. 02-01, 2002.

${ }^{4}$ O'Rourke, J., Computational Geometry in C, $2^{\text {nd }}$ Edition, Cambridge University Press, 2001.

${ }^{5}$ Held, M., "VRONI: An engineering approach to the reliable and efficient computation of Voronoi diagrams of points and line segments," Computational Geometry, Vol. 18, pp. 95-123, 2001.

${ }^{6}$ Legarth, B., "Debonding of Particles in Anisotropic Materials," International Journal of Mechanical Sciences, Vol. 45, pp. 1119-1133, 2003. 